\title{
Incidence of bleeding in children undergoing circumcision with ketorolac administration
}

\author{
Bruce Gao ${ }^{1}$; Taylor Remondini ${ }^{1}$; Navraj Dhaliwall ${ }^{1}$; Adrian Frusescu $^{2}$; Premal Patel $^{3}$; Anthony \\ Cook $^{4}$; Carolina Fermin-Risso ${ }^{4}$; Bryce Weber ${ }^{4}$ \\ ${ }^{1}$ Undergraduate Medicine, University of Calgary, Calgary, AB; ${ }^{2}$ Undergraduate Nursing, University of Calgary, \\ University of Calgary, Calgary, AB; ${ }^{3}$ Department of Surgery, University of Manitoba, Winnipeg, MB; ${ }^{4}$ Department \\ of Surgery, Alberta Children's Hospital, Calgary, AB; Canada
}

Cite as: Can Urol Assoc J 2018; Epub ahead of print. http://dx.doi.org/10.5489/cuaj.4632

\section{Published online December 1, 2017}

\section{*** \\ Abstract}

Introduction: Circumcision is the most common surgical procedure performed by pediatric urologists. Ketorolac has been shown to have an efficacy similar to morphine in multimodal analgesic regimens without the commonly associated adverse effects. Concerns with perioperative bleeding limit the use of ketorolac as an adjunct for pain control in surgical patients. As such, we sought to evaluate our institutional outcomes with respect to ketorolac and postoperative bleeding.

Methods: We retrospectively reviewed all pediatric patients undergoing circumcision from January 1, 2014 to December 31, 2015 at the Alberta Children’s Hospital. Demographics, perioperative analgesic regimens, and return to emergency department or clinic for bleeding were gathered through chart review.

Results: A total of 475 patients undergoing circumcisions were studied, including 150 (32\%) who received perioperative ketorolac and 325 (68\%) who received standard analgesia. Patients receiving ketorolac were more likely to return to the emergency department or clinic for bleeding (ketorolac group 19/150 [11\%], non-ketorolac group 16/325 [3.3\%]; p=0.005). Patients receiving ketorolac were more likely to have postoperative sanguineous drainage (ketorolac group 96/150 [64.0\%], non-ketorolac group 150/325 [46.2\%]; $\mathrm{p}<0.001$ ). There was no significant difference in the number of patients requiring postoperative admission or further medical intervention.

Conclusions: Although a promising analgesic, ketorolac requires additional investigation for safe usage in circumcisions due to possible increased risk of bleeding. 


\section{Introduction}

Circumcision is a common surgical intervention in pediatric urology indicated in instances of refractory balanitis, pathological phimosis, or paraphimosis. The control of post-operative pain is essential in order to decrease patient morbidity and to improve patient and parent satisfaction. Methods to reduce post-operative morbidity in circumcision include the use of general anesthesia combined with regional block. Common regional blocks include dorsal penile nerve blocks (DPNB) or caudal epidural blocks (CB). Neither has been shown to be more effective than the other in terms of the need for rescue or other analgesia. ${ }^{1,2}$ DPNB may be preferred over CB in the case of ambulatory children as CB carries the risk of temporary leg weakness after use.

Even with current analgesia standards, pediatric surgical patients are often at risk of being undertreated for postoperative pain when the regional anesthesia resolves. ${ }^{3}$ Pain is by far the most common complaint of parents after circumcision ${ }^{2}$ and a common reason for parents to avoid pursuing circumcision for their child. ${ }^{4}$ Ketorolac is an effective nonsteroidal antiinflammatory drug (NSAID) that provides an analgesic effect through inhibition of COX-1 and COX-2 within the body. ${ }^{5}$ Compared to opioids, ketorolac offers equivalent levels of pain relief with a decreased incidence of postoperative nausea and vomiting. ${ }^{6,7}$ A single dose of systemic ketorolac has been demonstrated to be an effective adjunct in multimodal analgesia regimens in order to reduce post-operative pain. ${ }^{8}$

Although ketorolac is a promising analgesic, trials for tonsillectomies have suggested an increased risk of post-operative bleeding. ${ }^{9,10}$ This finding however is not consistent. ${ }^{6}$ The fear of bleeding has lead many otolaryngologists and anesthesiologists avoid ketorolac in tonsillectomies and other conditions that may have an increased risk of bleeding. Bleeding, although uncommon, is the most common complication suffered by these children receiving ketorolac. ${ }^{10}$ Overall, the favorable side effect profile of ketorolac is balanced by concerns of post-operative bleeding. With circumcision being a high volume pediatric urological procedure, we sought to evaluate the bleeding outcomes of children receiving ketorolac for circumcision.

\section{Methods}

We conducted a retrospective study of pediatric patients ( $<18$ years old) at the Alberta Children's Hospital who underwent circumcision for refractory balanitis, pathological phimosis, paraphimosis, penile torque or hypospadias from January 1st, 2014 to December 31st, 2015. Only charts of patients undergoing circumcision for refractory balanitis, pathological phimosis and paraphimosis were included. The retrospective review spanned operative notes, nursing care notes, emergency charts and clinic follow-ups of each patient. Demographics, peri-operative analgesic regimens, and bleeding events in post-operative care, emergency department and clinic were gathered through chart review. Three incomplete charts were excluded and all information was anonymized. Two databases were cross referenced (one provided by anesthesia and another by health records) to ensure that charts were congruent and complete.

Informed consent was obtained from all parents after an explanation of the procedure, alternate options and possible risks. Three surgeons were performing the procedures at the time 
and surgical and anesthetic technique were similar in all cases. Based on the preference of the anesthetist and surgeon, children received either a standard analgesic regimen or a regimen augmented with intravenous ketorolac $(0.5 \mathrm{mg} / \mathrm{kg}$, maximum $30 \mathrm{mg})$. All children received a 15 $\mathrm{mg} / \mathrm{kg}$ oral dose of acetaminophen prior to surgery unless contraindicated. General anesthesia was induced with sevoflurane via facemask. After establishing venous access, a laryngeal mask was inserted, and anesthesia was maintained with 1 minimum alveolar anesthetic concentration (MAC) of sevoflurane in oxygen/air 50/50 mixture. The DPNB nerve block was performed by a pediatric urologist using a $23 \mathrm{GA}$ needle inserted below the Buck fascia. Once the needle tip was positioned appropriately, and after a negative aspiration test, $0.2 \mathrm{~mL} / \mathrm{kg}$ (maximum $10 \mathrm{~mL}$ ) of $0.25 \%$ bupivacaine was injected in small aliquots, with intermittent aspiration throughout. In all patients, skin incision was performed at least 5 min after placement of the nerve block. At the beginning of the circumcision procedure under general anesthetic, patients in the ketorolac group received a $0.5 \mathrm{mg} / \mathrm{kg}$ (maximum $30 \mathrm{mg}$ ) intravenous dose of ketorolac. Patients were advised to take ibuprofen and acetaminophen post-operatively as needed, but not ibuprofen within 6 hours of the procedure due to increased bleeding risk. Noninvasive blood pressure monitoring, electrocardiography monitoring and pulse oximeter were routinely used.

The surgical technique was similar in all cases - an excision technique with use of an artery clamp. ${ }^{11}$ After being prepped and draped in supine position, the phimotic ring was stretched and preputial adhesions were bluntly dissected. Excess foreskin was clamped by an artery clamp then transected with monopolar cautery. Bipolar cautery was used to obtain good hemostasis. 5-0 Vicryl Rapide was used to reapproximate the foreskin. Dermabond glue was used and polysporin applied. The patient was then transferred to the postanesthetic care unit (PACU). No dressings were applied to the surgical site.

After the procedure, children were observed in the PACU by a nurse. If necessary, analgesics (acetaminophen) were given by a member of the anesthesia team. Children were discharged from the hospital after a period of observation and spontaneous micturition. Statistical analysis via chi-square was performed using SPSS analytical software.

\section{Results}

Patient demographics are shown in table 1. Between the two year period (January 1st, 2014 to December 31st, 2015), 475 children 5 days to 17 years old (mean age $5.3+/$ - 4.2) underwent circumcision at the Alberta Children's Hospital. A general analgesic regimen was used in 325 children (68\%), while peri-operative ketorolac was used in 150 children (32\%). There was no significant difference in age between groups (ketorolac group: $5.6+/-4.1$, non-ketorolac group: $5.2+/-4.2, \mathrm{p}=0.496)$ and perioperative ketorolac was administered in the ketorolac group (0.44 $\mathrm{mg} / \mathrm{kg}+/-0.14 \mathrm{mg} / \mathrm{kg}$, range $0.11 \mathrm{mg} / \mathrm{kg}-1.14 \mathrm{mg} / \mathrm{kg}$ ).

Results of data analysis is displayed in table 2 . There was a significantly higher incidence of post-operative sanguineous drainage for ketorolac patients; $64.0 \%$ ketorolac vs $46.2 \%$ nonketorolac (OR = 2.07, 95\% CI 1.39 - 3.09; $\mathrm{p}<0.001)$. There was a significantly higher return to the emergency department or clinic for bleeding for ketorolac patients; $12.7 \%$ ketorolac vs $4.9 \%$ 
non-ketorolac ( $\mathrm{OR}=2.63,95 \% \mathrm{CI} 1.30$ - 5.32; $\mathrm{p}=0.005)$. There was no difference in postoperative admission between the groups, $0 \%$ ketorolac vs $0.9 \%$ non-ketorolac (OR 0.991 , 95\% CI 0.98 - 1.00; $\mathrm{p}=0.238$ ).

\section{Discussion}

Circumcision is the most common surgical procedure performed by pediatric urologists. Ketorolac has been shown to have an efficacy similar to morphine in multi-modal analgesic regimens without the commonly associated adverse effects. ${ }^{6,7}$ Unfortunately, the most common side effect of ketorolac is increased bleeding. ${ }^{5}$ As such the objective of this study was to retrospectively evaluate bleeding outcomes in children undergoing circumcision with ketorolac. In addition, any serious adverse events would help in the design of future randomized trials involving ketorolac in pediatric surgeries.

Overall, there was a significantly higher incidence $(\mathrm{p}<0.001)$ of post-operative sanguineous drainage for ketorolac patients $(96 / 150,63.7 \%)$ compared to the non-ketorolac group (150/325, 46.3\%). In other words, the surgical site of ketorolac patients looked bloodier than those of standard analgesia patients. With ketorolac patients exhibiting a bloodier surgical site, surgeons may be called to the post-anesthetic care unit (PACU) more frequently. In addition, patients receiving ketorolac were found to be more likely to return to the emergency department or clinic for bleeding (ketorolac group - 19/150, 12.7\%, non-ketorolac group $16 / 325,4.9 \%, p=0.005)$. This suggests a possible safety concern towards ketorolac administration in pediatric patients. Furthermore, additional visits to a physician increase the burden to our healthcare system. There was no significant difference for post-operative admission between groups. Although promising, the low incidence of admissions makes this result inconclusive.

An attempt was made to evaluate a dose-response relationship between ketorolac and risk of bleeding. However, anesthetists at the Alberta Children's Hospital tended to use an average $0.5 \mathrm{mg} / \mathrm{kg}$ dosage of ketorolac for each patient and therefore no dose response was seen. Furthermore, the number of patients with post-operative bleeding requiring return to medical attention was low, making this a small sample size to evaluate.

There are currently no studies regarding circumcision and ketorolac in previous literature to our knowledge. That being said, pediatric tonsillectomy studies have demonstrated increased risk of bleeding with ketorolac ${ }^{9,10}$. This finding however is not consistent. ${ }^{6}$ Although findings in literature are conflicting, the fear of bleeding has lead many otolaryngologists and anesthesiologists avoid ketorolac in tonsillectomies and other conditions that may have an increased risk of bleeding. The results of this circumcision study raises concerns about bleeding and supports the findings of most pediatric tonsillectomy studies,

As a retrospective cohort study, this study was limited in a few areas. Firstly, key outcomes such as post-operative pain control were not recorded. This means that although ketorolac resulted in more bleeding events, we have no indication of whether the pain control may have been worth the risk. To further explore this area, a randomized controlled trial of pain 
control in ketorolac and non-ketorolac patient is currently underway. In addition, rare outcomes such as post-op admission or additional surgical intervention were difficult to evaluate. A meaningful conclusion for these outcomes would require a much larger sample size than the number of studies that we had for review at the Alberta Children's Hospital.

Another limitation was that patients could have presented to hospitals other than the Alberta Children's Hospital for bleeding. As a result, some bleeding cases could have been missed. To reduce this effect, patients were given a standardized sheet that instructed them to come back to Alberta Children's Hospital given any concerns. However, this may not have always been followed by patients. In addition, patients were instructed to take Advil and Tylenol for post-operative pain and unfortunately, adding an NSAID could have increased the anticoagulation effect. With this database, it was not possible to tell which patients took Advil or how much they took. An ongoing prospective study with post-operative home phone calls aims to evaluate this parameter further.

Overall, ketorolac patients had significantly more bleeding events requiring medical attention than patients receiving standard analgesia during circumcision. In addition, surgeons may be called to the PACU more frequently with more sanguineous ooze noted in ketorolac patients. Although a promising analgesic, ketorolac requires additional investigation for safe usage in circumcisions due to possible increased risk of bleeding. We have launched a randomized controlled trial evaluating the pain control and safety of ketorolac in pediatric circumcisions and hope to provide more answers on this topic. 


\section{References}

1. Cyna AM, and Middleton P. "Caudal epidural block versus other methods of postoperative pain relief for circumcision in boys." Cochrane Database of Systematic Reviews. 4 (2008): CD003005.

2. Brady-Fryer B. et al. "Pain relief for neonatal circumcision." Cochrane Database of Systematic Reviews. 4 (2004): CD004217.

3. Apfelbaum JL, et al. "Practice guidelines for acute pain management in the perioperative setting: an updated report by the American Society of Anesthesiologists Task Force on Acute Pain Management.” Anesthesiology 116.2 (2012): 248-273.

4. Adler R, Ottaway MS, and Gould S. "Circumcision: we have heard from the experts; now let's hear from the parents.” Pediatrics 107.2 (2001): E20.

5. Ketorolac compound summary. Pubchem Substance Website. National Center for Biotechnology, US National Library of Medicine. http://pubchem.ncbi.nlm.nih.gov/compound/3826. Accessed June 24, 2015.

6. Sutters KA, et al. "Analgesic efficacy and safety of single-dose intramuscular ketorolac for postoperative pain management in children following tonsillectomy.” Pain 61.1 (1995): 145-153.

7. Gunter JB, et al. "Recovery and complications after tonsillectomy in children: a comparison of ketorolac and morphine.” Anesthesia and Analagesia 81.6 (1995): 11361141.

8. De Oliveira GS Jr, Agarwal D, and Benzon HT. "Perioperative single dose ketorolac to prevent postoperative pain: a meta-analysis of randomized trials.” Anesthesia and Analgesia 114.2 (2012): 424-433.

9. Rusy LM, et al. "A double-blind evaluation of ketorolac tromethamine versus acetaminophen in pediatric tonsillectomy: analgesia and bleeding." Anesthesia and Analgesia 80.2 (1995): 226-229.

10. Splinter WM, et al. "Preoperative ketorolac increases bleeding after tonisillectomy in children” Canadian Journal of Anaesthesiology 43.6 (1996): 560-563.

Weiss HA, et al. "Complications of circumcision in male neonates, infants and children: a systematic review” BMC Urology 10.2 (2010).

11. Abdulwahab-Ahmed, A. \& Mungadi I. “Techniques of male circumcision” Journal of Surgical Technique \& Case Report. 5.1 (2013). 
Figures and Tables

Table 1. Patient demographic information in children undergoing circumcision with or without ketorolac administration

\begin{tabular}{|l|c|c|c|}
\hline & Overall & Ketorolac & Non-ketorolac \\
\hline Number of patients (all males) & 475 & 150 & 325 \\
\hline Age in years, M (SD) & $5.3(4.2)$ & $5.6(4.1)$ & $5.2(4.2)$ \\
\hline Ketorolac dosage in mg/kg, M (SD) & N/A & $0.44(0.14)$ & 0 \\
\hline
\end{tabular}

SD: standard deviation.

Table 2. Comparative analysis with chi-square for children undergoing circumcision with or without ketorolac administration

\begin{tabular}{|l|c|c|c|c|}
\hline & $\begin{array}{c}\text { Absolute count } \\
\text { without ketorolac }\end{array}$ & $\begin{array}{c}\text { Absolute count } \\
\text { with ketorolac }\end{array}$ & $\begin{array}{c}\text { Odds ratio (95\% } \\
\text { CI) }\end{array}$ & $\mathbf{p}$ \\
\hline $\begin{array}{l}\text { Postoperative } \\
\text { sanguineous drainage }\end{array}$ & $150 / 325(46.2 \%)$ & $96 / 150(64.0 \%)$ & $2.07(1.39-3.09)$ & $\mathbf{p}<\mathbf{0 . 0 0 1}$ \\
\hline $\begin{array}{l}\text { Return to ED or clinic for } \\
\text { bleeding }\end{array}$ & $16 / 325(4.9 \%)$ & $19 / 150(12.7 \%)$ & $2.63(1.30-5.32)$ & $\mathbf{p}=\mathbf{0 . 0 0 5}$ \\
\hline $\begin{array}{l}\text { Postoperative admission } \\
\text { within 30 days }\end{array}$ & $3 / 325(0.9 \%)$ & $0 / 150(0.0 \%)$ & $0.991(0.98-1.00)$ & $\mathrm{p}=0.238$ \\
\hline
\end{tabular}

CI: confidence interval; ED: emergency department. 